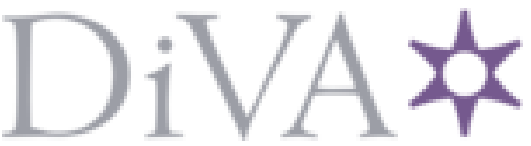

http://www.diva-portal.org

Preprint

This is the submitted version of a paper published in International Journal of Network Management.

Citation for the original published paper (version of record):

Kreuger, P., Steinert, R., Görnerup, O., Gillblad, D. (2018)

Distributed dynamic load balancing with applications in radio access networks

International Journal of Network Management, 28(2)

https://doi.org/10.1002/nem.2014

Access to the published version may require subscription.

N.B. When citing this work, cite the original published paper.

Permanent link to this version:

http://urn.kb.se/resolve?urn=urn:nbn:se:ri:diva-32825 


\title{
Distributed dynamic load balancing with applications in radio access networks
}

\author{
Per Kreuger*, Rebecca Steinert, Olof Görnerup and Daniel Gillblad \\ Swedish Institute of Computer Science (Rise SICS). Email: \{piak, rebste, olofg, dgi \}@sics.se
}

\begin{abstract}
SUMMARY
Managing and balancing load in distributed systems remains a challenging problem in resource management, especially in networked systems where scalability concerns favour distributed and dynamic approaches. Distributed methods can also integrate well with centralised control paradigms if they provide high-level usage statistics and control interfaces for supporting and deploying centralised policy decisions. We present a general method to compute target values for an arbitrary metric on the local system state, and show that autonomous rebalancing actions based on the target values can be used to reliably and robustly improve the balance for metrics based on probabilistic risk estimates. To balance the trade-off between balancing efficiency and cost, we introduce two methods of deriving rebalancing actuations from the computed targets that depend on parameters which directly affects the trade-off. This enables policy level control of the distributed mechanism based on collected metric statistics from network elements. Evaluation results based on cellular RAN simulations indicate that load balancing based on probabilistic overload risk metrics provide more robust balancing solutions with fewer handovers compared to a baseline setting based on average load. Copyright (c) 0000 John Wiley \& Sons, Ltd.
\end{abstract}

Received ...

KEY WORDS: Self-organising heterogeneous networks; Distributed dynamic load balancing; Methods/control theories; Network Management/Wireless \& mobile networks

\section{INTRODUCTION}

Distributed algorithms and self-organisation have been successfully employed for many network management tasks, e.g. routing, service discovery, failure recovery, and often exhibit clear scalability and reliability advantages. However, for effective control and coordination of infrastructure resources, the trend in modern network management tends more toward (logically) centralised solutions implemented in programmable networking environments. Although a centralised management paradigm better support optimised resource management and service deployment strategies, real-time systems and services still depend on highly distributed management functions for scalable and timely networking operations. One fundamental design challenge in future management of networked systems involves finding a balance between distributed and centralised network operations, another identifying high-level abstractions for controlling and representing the state of heterogeneous infrastructures.

We propose a generic approach to dynamic balancing of resources in networked systems that adresses these challenges based on the notion of Distributed Target Computation (DTC) [1,2] operating on probabilistic risk metrics. We introduce risk as a metric for representing the probability

${ }^{*}$ Correspondence to: Rise SICS, Box 1263, SE-164 29 Kista, Sweden. Email: piak@ sics . se 
of over-consuming a user-specified fraction of a given resource. Risks are examples of high-level abstractions needed for centralised decisions, while parameters in the metrics and rebalancing tactics open up for centralised control of essentially distributed mechanisms. In a network setting, metrics and targets are computed locally at individual network entities (or nodes) using information from neighbouring entities. In the case of load balancing, a probabilistic risk metric relates to the risk of overloading a serving network entity (e.g. an access point).

The main benefits of DTC include simplicity, scalability and robustness. DTC is naturally distributed in the sense that the metrics and targets are computed locally by, or for, each single node using only information obtained from other nodes in its neighbourhood. The benefit of balancing of a probabilistic metric entails a unified representation and an abstraction of the network state and capacity, in contrast to dealing with vendor specific metrics that are often varying in range and are difficult to compare. Additionally, probabilistic risk estimates accounts for variability in the observations which leads to a more robust actuator and balancing mechanism. Although we focus mainly on load balancing in the context of Radio Access Networks (RAN), the approach is applicable to a range of resource management applications (e.g. in cloud systems and traffic management) and resource metrics, (e.g. compute power, buffer memory and/or node connectivity).

\subsection{Contribution}

In [1], we presented a basic DTC in the context of LTE using one particular target load metric and a specific way of producing the Cell Range Expansion (CRE) bias values. That method proved to be very effective, but relatively insensitive to cost incurred by the CRE changes. In this paper, we address this issue by using probabilistic risk of high-load levels as an alternative metric, and a generalised interface for controlling rebalancing actions, based on the expanded exposition of the principles of the balancing approach.

The core contribution is the generic algorithm and modelling principles of the distributed balancing approach. The applicability of the approach is evaluated in a cellular LTE setting and further exemplified for WiFi systems. The generality and uniformity of the proposed metrics make them ideal also for centralised management in virtualised controllers in a multi-RAT scenario.

More specifically, the main contributions of this paper are:

- A general method for dynamically balancing a metric between nodes in a distributed system

- Application of the method to balance radio resource load in RANs using probabilistic risk estimates and a remotely configurable rebalancing mechanism.

After a short overview of relevant state of the art, we will present DTC in full generality, then specialise and evaluate it for the RAN load balancing problem. This is followed by a discussion and conclusions.

\section{RELATED WORK}

Load balancing is used in several areas outside networking, e.g. to plan resource allocation in data centers, work schedules and industrial production, as well as some in networking not touched upon here, e.g. routing. In some of these cases, centralised decisions $[3,4]$ and static methods $[5,6]$ are feasible. For others, including many in networking, dynamic methods $[4,7]$ and distributed decisions $[8,9]$ are more suitable.

Mobility balancing for cellular networks is a well studied topic. Early proposals for cellular RAN include [10] which employed adaptive cell sizing through cell transmit power regulation (physical power "breathing") to offload UEs from highly loaded cells, and similar approaches has more recently been proposed [11] for WiFi. The CRE mechanisms in LTE allows a similar rebalancing actuations, but without the complexity of physical power management.

Another distributed approach is described by Chen [12], where it is used for negotiating area coverage between base station agents. It is based on bilateral exchanges between base stations, but 
use complex cost/benefit estimates for each of a large number subareas coverable by several cells. Base stations negotiate exchanges of subareas to maximise benefit over cost in a two-step multiagent process, and synchronously commit to the most favourable ones. By contrast our approach never modifies physical coverage, but only the CRE bias parameter, and exchanges between nodes implement a rapid gradient descent involving all neighbours of a single node instead of a multi-stage agent bidding process.

Several authors propose physically centralised solutions, e.g. Lobinger et al. [13] who report work where load balancing is performed by considering individual user "satisfaction" based on both SINR and node radio load. They propose a method based on selecting individual candidate UEs and target-cells for off-loading, evaluating the effect on total "satisfaction", before committing to each handover. Another centralised approach [14] by Siomina and Yuan introduces a method based on integer programming, to assign CRE values to each node, given load levels of the entire network. The method requires collecting and transferring load estimates to a central location, where a potentially time consuming optimisation mechanism can determine suitable values for the CRE parameters. It is unclear how the authors intend to handle the delays and scalability issues implied by such a method. Similar issues arise in the centralised approach described in [15] which uses enforced handovers rather than manipulation of the CRE parameter.

Hao et al. [16] propose managing individual handovers which has the potential to improve balance more efficiently than physical breathing, CRE based methods, or indeed any global method treating UEs as a collective. As in the case of [13], this comes at a considerable cost in terms of management overhead and computational complexity.

Our approach is based on the methods presented in [1] but introduces balancing over-load risk, and an actuation mechanism based on a parametrised sigmoid mapping. These are useful as a state abstractions, and as controller APIs for logically centralised control within a unified management paradigm for heterogeneous infrastructures.

From a programmability perspective several approaches and architectures for flexible management of software-defined RANs have been proposed [17-19]. The proposed architectures are aimed to enable logically centralised control for dynamic resource management and RANslicing [17]. Moreover, this paradigm allows for effective coordination of radio resources and interference management. A logically centralised controller plane may consist of various types of controllers capable of operating at different time-scales in order perform control operations both in real-time and at longer time-scales $[17,19]$. However, scalability of centralised approaches remains an issue.

The concept of programmability abstractions [20], (here realised as load metrics) is central for effective coordination and control of heterogeneous radio infrastructures and goes hand-in-hand with the development of architectures for software-defined RANs. The probabilistic load metrics proposed in our work are instances of such abstractions. The combination of logically centralised control and abstract representation of the network state enables automated and dynamic resource allocation fulfilling specified service and user requirements. Since load balancing in general is fundamental to ensure bandwidth and delay requirements, abstractions representing risk of overload is highly relevant to proactively avoid service unavailability and performance degradations.

\section{METHOD}

We represent a dynamic system as a graph (Figure 1) where nodes constitute resources with an associated usage or load state, encoded as a single value $0 \leq m_{i} \leq 1$, its metric, and edges represent neighbourhood relations. In the RAN case neighbourhoods correspond to cells with overlapping coverage, but although the complexity of the computation depends on the connectivity of the graph, in principle any binary relation is admissable.

How the usage state is generated does not concern us at this point, but we assume we can influence it through an actuation method parametrised by local bias values derived as the difference between the usage metric and a corresponding target value $t_{i}$ computed for each node by DTC. 


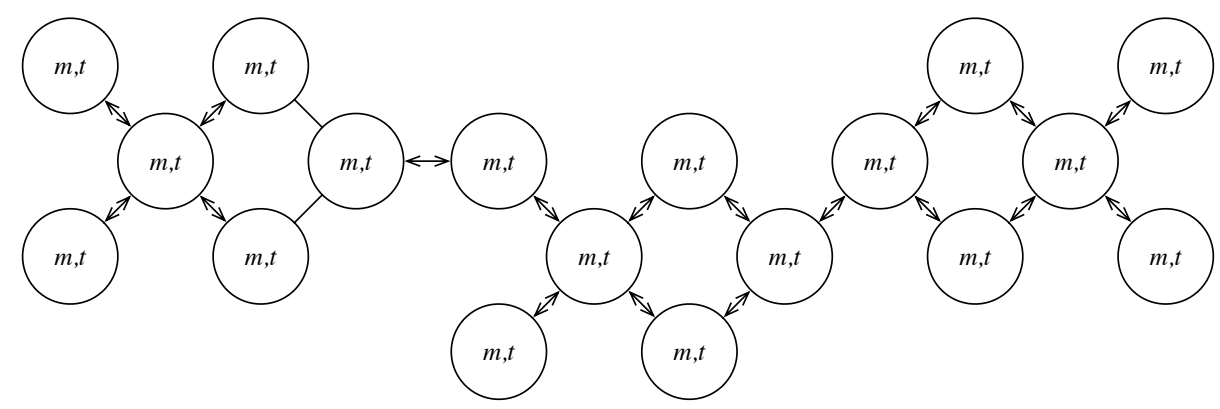

Figure 1. Example system graph. The system state is represented by the metric and target values associated with the nodes of the graph. State transitions are either metric updates or recomputation of the DTC targets.

We first present the method in a form where the balancing metric $m_{i}$, is left as a parameter, and assume that the rebalancing mechanism takes a single actuation bias value per node as input, but for generality, use a function $\mathcal{B}$, to derive that value from the node state. For any concrete balancing problem $m_{i}$ and $\mathcal{B}$ must be defined separately, and we will do so in full detail for the RAN load balancing scenario in Section 4. In summary, we presume the following:

\subsection{Prerequisites}

1. A way for each node to identify a neighbourhood of other nodes.

2. A current metric value $m_{i}$ for each node $i$.

3. A mechanism for nodes to exchange target and metric values bilaterally.

4. A mechanism to influence the choice between nodes to serve client demands.

\subsection{Local neighbourhoods}

We maintain for each node a local neighbourhood, i.e. a list of related nodes with which it exchanges target and metric values. The neighbourhood can be either preconfigured or built up and updated dynamically based on observed node interactions, e.g. (following a scheme introduced in [21,22]):

Model the probability $p_{i j}$ of each node $i$ having an interaction with another node $j$ by an estimate $\hat{p}_{i j}$. Assign for each node $j$ in a set $j \in J_{i}$ of nodes with which $i$ can be expected to have interactions ${ }^{\dagger}$, a prior

$$
\hat{p}_{i j}^{\prime}=\frac{1}{\left|J_{i}\right|}
$$

and initialise interaction counters $c_{i j}=0$ and $c_{i}=0$. Increment for each observed interaction between $i$ and $j$, counters $c_{i j}$ and $c_{i}$, and estimate the probability of an interaction between $i$ and $j$ as follows:

$$
\hat{p}_{i j}=\frac{w \hat{p}_{i j}^{\prime}+c_{i j}}{w+c_{i}}
$$

where $w$ is a weight controlling the decay rate of the estimate. Here, $\hat{p}_{i j}$ represents the expectation of the posterior distribution assuming a Dirichlet prior with parameters $w p_{i j}$ and observation counts $c_{i j}$. In addition, replace all priors by the estimated posteriors, $\hat{p}_{i j}^{\prime}=\hat{p}_{i j}$, and reset counters $c_{i}$ and all $c_{i j}$ after a fixed number of observations. This gives a smooth exponential decay of the influence of older observations while ensuring that the estimates for all $j \in J_{i}$ sum to 1 .

Finally, let the local neighbourhood $\mathcal{N}_{i}$ be the smallest subset of $J_{i}$ such that

$$
\sum_{j \in \mathcal{N}_{i}} \hat{p}_{i j}>n
$$
${ }^{\dagger}$ It is not crucial that $J_{i}$ initially include all possible nodes, since any one $l \notin J_{i}$ can be added as needed by creating a
new counter $c_{i l}$, and assigning (at the cost of a negative initial bias) to it, a prior $\hat{p}_{i l}^{\prime}=0$. 
for some minimum value $n$ on the joint probability of an interaction with a node in $\mathcal{N}_{i}$, and so that, for any $l \in J_{i} \backslash \mathcal{N}_{i}$, for all $j \in \mathcal{N}_{i}, \hat{p}_{i l} \leq \hat{p}_{i j}$. $\mathcal{N}_{i}$ will thus, according to the estimates, consist of the most likely nodes $j \in J_{i}$ for $i$ to have interactions with.

\subsection{Metrics}

The type of momentary measurement statistics used to derive the metrics will differ with the application, but generally we expect the metrics to be probabilistic estimates produced from periodic observations of some variable closely related to the balancing objective. We assume that it is updated regularly and is available to the DTC update mechanism at any time.

We will see several examples of concrete metrics in Section 4.1.1, but for this formulation of DTC, the details of how the $m_{i}$ are produced or collected, is not essential.

\subsection{Distributed target computation}

We update the target value for each node triggered by the detection of some event at the node. The event can be either a timeout, a significant change in the metric value, an interaction with another node, or requested by an external mechanism. The computation of target values is distributed between the nodes in the network, but any individual node interacts only with the nodes in its neighbourhood. Computation of target values can be done by either polling or pushing target values between neighbours. We will describe an on-demand polling version of the computation, but it is not difficult to imagine an asynchronous push variation, which may be preferable for some applications.

3.4.1. Local target update Whenever a node $i$ needs to update its current target $t_{i}$, it executes the following procedure:

1. Retrieve, for each node $j \in \mathcal{N}_{i}$ their current target values $t_{j}$

2. Adjust $i$ 's target value $t_{i}$ to the mean of the local metric value $m_{i}$ and the previous target values $t_{j}$ :

$$
t_{i}^{n}=\frac{m_{i}+\sum_{j \in \mathcal{N}_{i}} t_{j}^{n-1}}{1+\left|\mathcal{N}_{i}\right|}
$$

3. Request that all $j$ in $i$ 's neighbourhood $\mathcal{N}_{i}$ adjust their targets $t_{j}$ as in Step 2.

4. If $t_{i}$ differs from its previous value by less than a cutoff value, terminate the procedure.

5. If not, and unless a maximum number of iterations have been reached, go to Step 1.

3.4.2. Convergence The update operation for a single node $i$ iterates over $i$ and $\mathcal{N}_{i}$, interleaving adjustments of their target values $t_{i}$ and $t_{j}$ for $j \in \mathcal{N}_{j}$. Provided that the metrics $m_{i}$ and all $m_{j}$ for $j \in \mathcal{N}_{i}$ remain fixed, and that updates of the $t_{j}$, consider only target changes within $\mathcal{N}_{i}$, each involved node adjusts at each step its target to reduce the value of a convergence metric

$$
\left|t_{i}-\frac{m_{i}+\sum_{j \in \mathcal{N}_{i}}\left|t_{j}-\frac{m_{j}+\sum_{k \in \mathcal{N}_{j}} t_{k}}{1+\left|\mathcal{N}_{j}\right|}\right|}{1+\left|\mathcal{N}_{i}\right|}\right|
$$

Each application of Equation 4 to a node $i$ or one of the $j \in \mathcal{N}_{i}$ eliminates its direct contribution to the convergence metric of $i$, but may, due to the node's occurrence in neighbourhoods of other nodes within $\mathcal{N}_{i}$, increase those of other nodes. Repeated interleaved application will however eventually reduce the metric to zero. This is true for the node $i$ itself but not necessarily for all nodes in $j \in \mathcal{N}_{i}$ since their convergence metric may also depend on nodes outside $\mathcal{N}_{i}$.

The number of steps required for the convergence of a single node depends on the connectivity within its neighbourhood, but for typical HetNet scenarios, where only macro nodes have handovers with more than a few other nodes, the total number of iterations needed to reduce the convergence 
metric to less than $10^{-3}$ is rarely more than 10 . Within each iteration the number of messages is bounded by $\left|\mathcal{N}_{i}\right|^{2}$ but is typically closer to $\left|\mathcal{N}_{i}\right| \log \left(\left|\mathcal{N}_{i}\right|\right)$.

If all nodes are updated regularly, and local metrics remain fixed, the entire system will eventually converge towards a global equilibrium where Equation 5 evaluates to zero for every node $k$, and the sum of all target to metric offsets, $\sum\left(t_{k}-m_{k}\right)$ is also zero. For a large, highly connected system, complete convergence may take considerable time, depending on the initial distance from the equilibrium state. On the other hand, the local improvment is immediate and often consideable, and in a dynamic system, where metrics change continuously, the system will always tend towards a target distribution that approximates the global eqilibrium. Typical performance of systems of up to 15 nodes are presented in section 5 .

The following section presents the update mechanism in more detail.

\subsubsection{Algorithm}

One step target adjustment Steps 1-2 of Section 3.4.1 are detailed in Algorithm 1: On receiving the message "adjustTarget(neighbourhood)" a node requests updated target values from its neighbours, then updates its local target value.

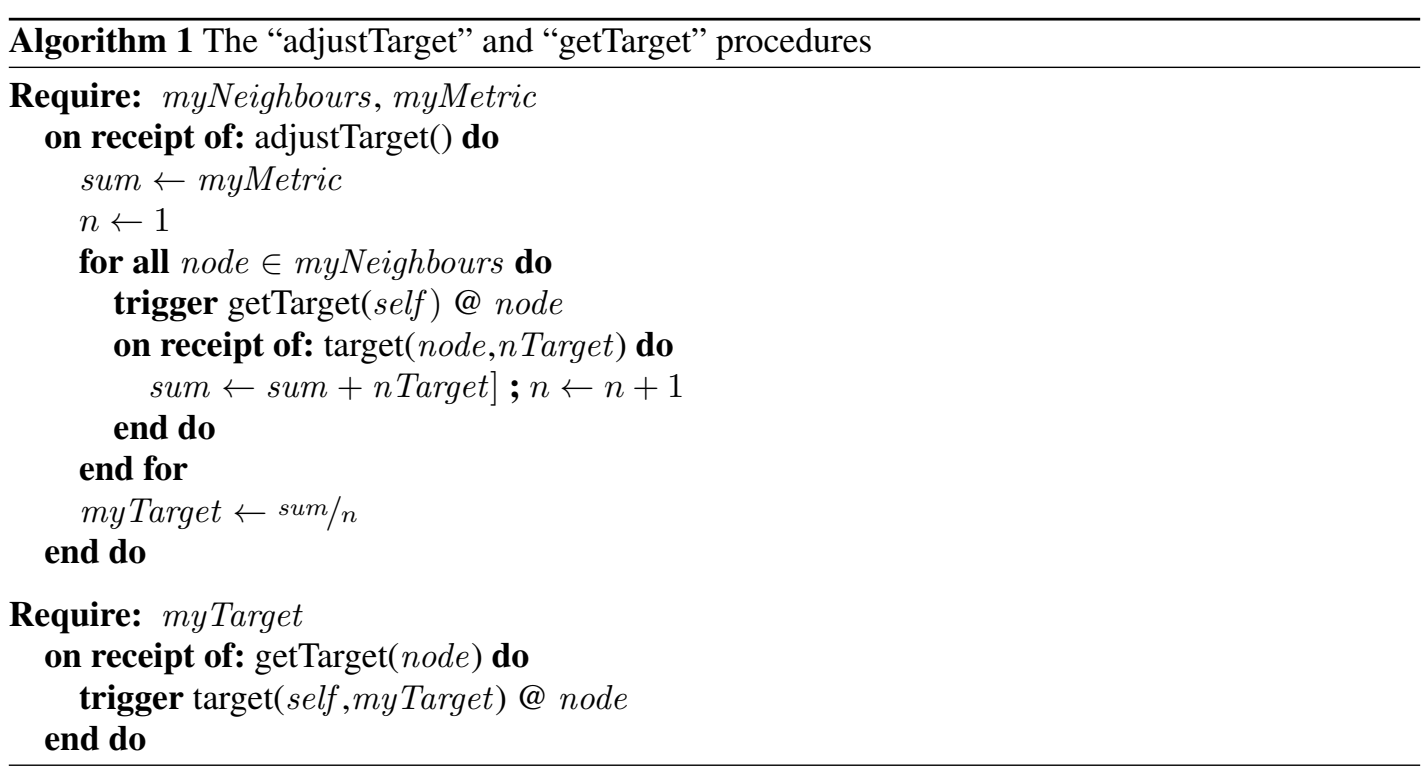

Neighbourhood-wide target update The complete local target update routine, including the iteration of steps 3-5, is illustrated in Algorithm 2: On receiving message "updateTarget()", a node first adjusts its own target value as in Algorithm 1, then request the nodes of its neighbourhood to do the same, but using updated targets only from within myNeighbours. If the resulting target value differs by more than cutoff from the previous one, the procedure is repeated.

\subsection{Biasing}

How we actuate a change in the balance of a chosen metric depends strongly on the application domain, but since we here consider primarily systems where distributed nodes serve client demands, we restrict the presentation to mechanisms which depend on a single actuation bias to skew the choice between nodes to serve clients and refer to a function mapping the node state to such a value as a biasing function. In principle any aspect of the state of a node and its neighbourhood can be used as input to the biasing function, but since DTC produces a local target to metric offset for each node, we will focus on biasing functions which takes these as inputs. The simplest biasing 


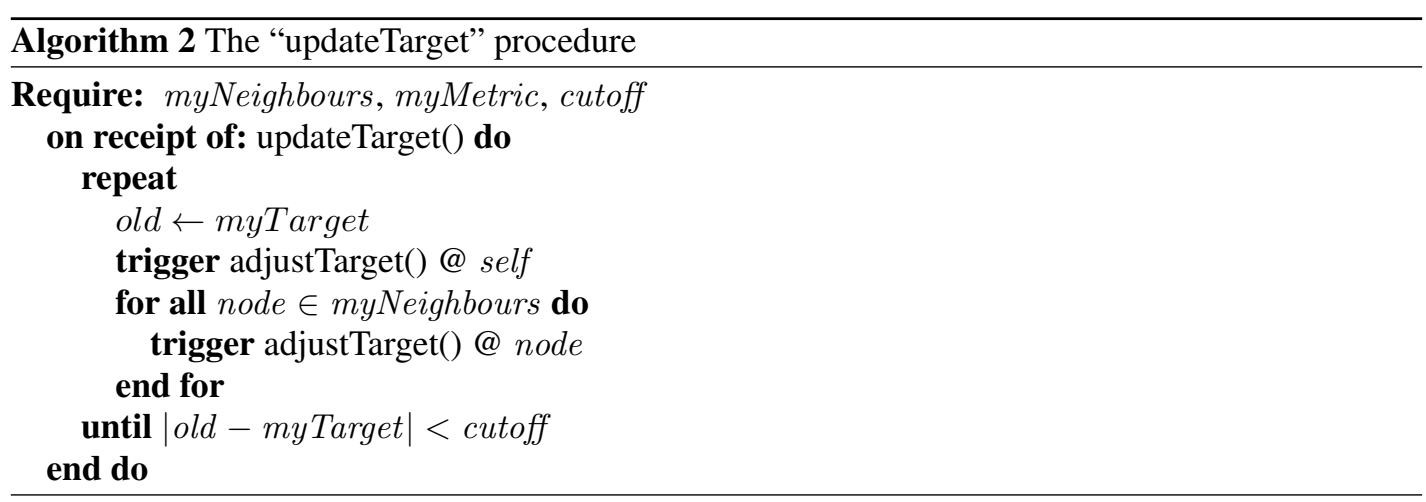

function just returns the local offset, but as we will see in Section 4, scaling the local offset, and using the distribution of offsets within the neighbourhood, can improve the actuation performance and reduce rebalancing cost. Algorithm 3 is thus parametrised by a biasing function $\mathcal{B}$, a set of control parameters and the local and neighbourhood offsets produced by Algorithms 1-2.



Algorithm 4 is an example code entry point for a node, assuming actuation reads and uses the bias produced by Algoritm 3. It initialises and updates the local target and bias, and then goes to sleep, awaiting the next update trigger.






\section{RAN LOAD BALANCING}

We will examine two network load balancing applications of which the cellular case (LTE) is by far the most detailed. The WiFi case is covered by a outline of one way a corresponding mechanism may be implemented.

\subsection{LTE load balancing}

In LTE the requirements of Section 3.1 can be fulfilled by fairly straight-forward extensions of existing mechanisms. Requirement 1 can be covered by simply pre-configuring the neighbourhood of each node at deployment time, or by implementing the method described in Section 3.2, based on simple handover counters for pairs of nodes. Requirement 3 needs a simple node-to-node communication protocol (e.g. an extension to the X2 protocol [23]) to allow requests for and reports of two high precision numbers, or a centralised mechanism that can emulate such exchanges for the nodes in a particular region. Once that is in place, the distributed computation of target values is straight-forward to implement, more or less directly as stated in Algorithms 1-2. Requirement 4 is fulfilled by the existing cell range expansion (CRE) parameters of LTE (and CDMA), and the standard handover mechanism, although several other biasing mechanisms may also be envisioned. Section 4.1.2 will discuss two based on dynamic CRE adjustments, but first we will examine some of the details of how to fulfil Requirement 2.

4.1.1. Load metrics In network load balancing we will always start out with measurements of some type of resource usage. The resource could be buffer memory, decoding, forwarding or compute resources, or in the case of RANs, the radio bandwidth resource. Here we will only consider the radio resource, but other and combined measures are certainly also possible. In the LTE case we will assume that we have access to momentary radio resource load measurements produced at a regular intervals (e.g. few times per second) in terms of the number of used resources blocks divided by the number of available resource blocks, i.e. a number between 0 and 1 . Since such measurements typically fluctuates very rapidly, and we expect to regulate load on time scales of several minutes, we will consider two types of probabilistic metrics based on statistics of the underlying measurements.

Load mean metric Define an exponentially decaying moving average

$$
\hat{l}_{k}=\frac{w \hat{l}_{k-1}+n \bar{l}_{k}}{w+n}
$$

where $\bar{l}_{k}=\frac{1}{n} \sum_{i=(k-1) n}^{k n-1} l_{i}$ is the sample mean of momentary loads $l_{i}$ for the estimation period, i.e. $(k-1) n \leq i<k n$ and $\hat{l}_{0}$ is a prior initialised to any number between 0 and 1 . We update the prior expectation $\hat{l}_{k-1}$ after a fixed number of observations $n$, so that $k=\lfloor i / n\rfloor$. Keeping $n$ (and/or $w)$ relatively large will tend to smoothen variations of the metric, and reduce handovers caused by rapid fluctuations of the momentary load. The advantage over a traditional exponentially weighted moving average (EWMA), where $n=1$, is that we have separate control over the size $n$ of the window over which we estimate, and the decay rate $1 / w$.

High load risk metric As a refinement, we will introduce an estimate of the risk of high loads using a moment estimation scheme where the first load moment is estimated as in Equation 6, and the second moment (variance) by a corresponding exponentially decaying moving variance estimate. Assuming a Gaussian-Whishart prior [24], the expectation of the posterior variance distribution and the corresponding exponentially moving variance estimate can be written as

$$
\hat{v}_{k}=\frac{w \hat{v}_{k-1}+\sum_{i=(k-1) n}^{k n-1}\left(l_{i}-\bar{l}_{k}\right)^{2}+\frac{w n}{w+n}\left(\hat{l}_{k-1}-\bar{l}_{k}\right)^{2}}{w+n}
$$


where $\bar{l}_{k}$ is again the sample mean of momentary loads, $\hat{v}_{k-1}$ and $\hat{v}_{k}$, the prior and posterior expectation of the variance respectively, and $\hat{l}_{k-i}$, the prior expectation of the mean, as obtained in Equation 6 above. The subexpressions $\sum_{i=(k-1) n}^{k n-1}\left(l_{i}-\bar{l}_{k}\right)^{2}$ represents sample variance over the estimation period, and $\frac{w n}{w+n}\left(\hat{l}_{k-1}-s_{k}\right)^{2}$ a term compensating for the decay of the mean estimate.

Using these two moment estimates we then estimate the parameters of a lognormal model of the load distribution of the node using the method of moments (MoM):

$$
\left\{\begin{array}{l}
\hat{\mu}_{k}=\ln \hat{l}_{k}-\frac{1}{2} \hat{\sigma}_{k}^{2} \\
\hat{\sigma}_{k}^{2}=\ln \left(1+\frac{\hat{v}_{k}}{\hat{l}_{k}^{2}}\right)
\end{array}\right.
$$

and use the CDF of the estimated distribution to extract a momentary risk estimate $\hat{c}_{k}$ of observing loads over a given fraction $\phi$ (e.g. 95\%) of the node capacity $r$.

$$
\hat{c}_{k}=P(L>\phi r)=\frac{1}{2}-\frac{1}{2} \operatorname{erf}\left[\frac{\ln \phi r-\hat{\mu}_{k}}{\sqrt{2} \hat{\sigma}_{k}}\right]
$$

A note on the choice of a lognormal model Lognormal empirical distributions are common in the telecom domain. E.g. link-level throughput measurement often fit well to lognormal distributions (see e.g. [25-27]). Although there is no a priori reason to assume that LTE radio cell load levels are also lognormal ${ }^{\ddagger}$, our simulator experiments appear to support this assumption - see Figure 2. The load generating process in the simulator is the combined effect of Pareto-fitted individual UE traffic variations and the UE mobility patterns. The momentary UE density distribution is a function of original UE "home" positions, and Levý-walk style (heavy-tailed flight distances [29]) movements. Even so, the resulting load distributions do conform well to lognormal distributions, at least up until the point where a significant part of the demand remains unfulfilled due to link-level congestion. This is a pattern that is familiar from studies of (fixed) link load measurements, where the distribution becomes bimodal at high loads, and where the secondary mode appears close to, but below, the resource capacity limit. In this study [27], we also found that the probability mass of the secondary peak is often approximated well by the mass of the tail of a fitted distribution that extends beyond the capacity of the resource, approximating the demand distribution.

\subsubsection{LTE biasing}

LTE CRE parameter biasing by maximal scaling To redistribute the load of the network towards the computed target $t_{i}$ for each node $i$, one way is to assign CRE bias values to each node in a suitable range (e.g. $[-3,3] \mathrm{dB})$ in a way which uses as much as possible of an available CRE range locally, while preserving correct gradients between the nodes within each neighbourhood.

We propose to do so by using the following bias function

$$
\mathcal{B}\left(b_{i},\left\{b_{j}\right\}_{j \in \mathcal{N}_{i}}, \text { range }\right)=\frac{\mid \text { range } \mid\left(b_{i}-\epsilon\right)}{\hat{b}-\check{b}-2 \epsilon}
$$

using only the $\max \hat{b}=\max \left\{b_{i}\right\} \bigcup\left\{b_{j}\right\}_{j \in \mathcal{N}_{i}}$ and $\min \check{b}=\min \left\{b_{i}\right\} \bigcup\left\{b_{j}\right\}_{j \in \mathcal{N}_{i}}$ of the neighbourhood offset distribution. The interval $[\breve{b}, \hat{b}]$ represents the range of offsets that we need to represent within the neighbourhood, and we use that to scale the corresponding local offset $b_{i}$. This has the advantage of using the entire range of acceptable CRE values, but tends to give large swings as the

\footnotetext{
$\ddagger$ Nor have we found any published analysis of cell level load distributions, either supporting or falsifying any such claim. Naboulsi's [28] otherwise exellent overview of mobile traffic analysis results and data sources has no mention of resource load distributions.
} 


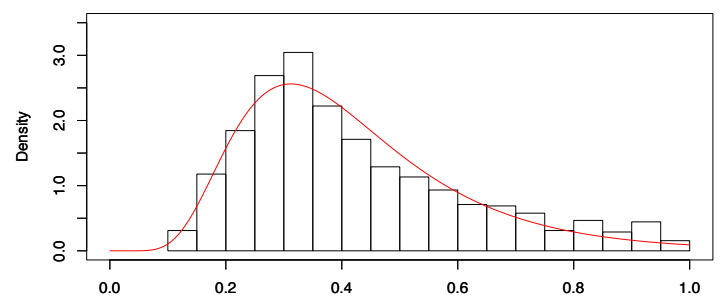

(a) Medium loaded macro cell

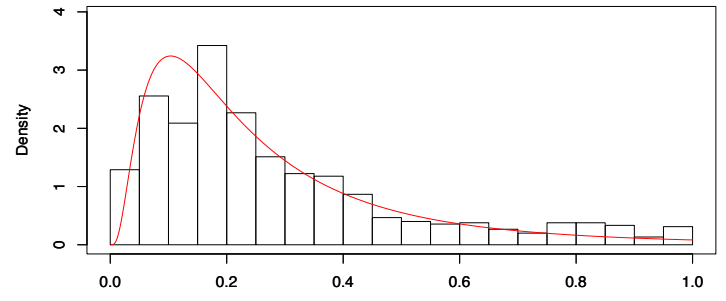

(b) Medium loaded small cell

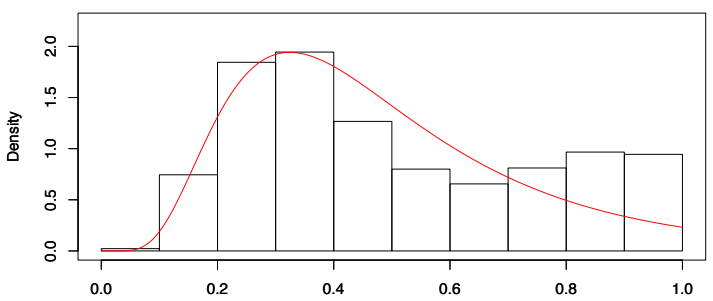

(c) Overloaded macro cell

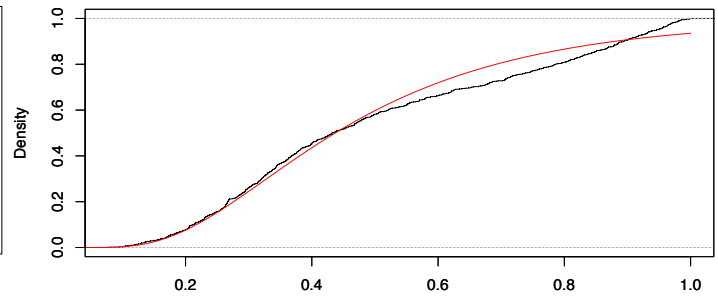

(d) CDF of above

Figure 2. Load distribution with lognormal fits for individual cells (random 3-minute extracts from simulator runs). Note that, although the empirical distribution in the right hand case is clearly bimodal, the value of the $\mathrm{CDF}$ of the fitted distribution in the region just below the cell capacity is in fact, quite accurate.

maximum difference in the neighbourhood approaches zero. We reduce this tendency by using a cutoff $\epsilon$ on $\hat{b}-\check{b}$, beyond which we avoid adjusting the CRE value.

As will be seen in Section 5, this biasing mechanism is very effective in terms of the balance achieved, but also costly in terms of handovers per connected user. Since it exploits the full range of CRE values there is also a high risk of serving some users with less than the optimal link quality.

Ideally we should actuate the rebalancing mechanism only when the gains to the overall performance of the system are significant, and only when it can be done at a reasonable cost to individual UE's channel quality. Switching to balancing risk of high loads is one step in this direction. Another is to scale the offset $b_{i}$ more smoothly, and so that only large differences in the metrics translates to large difference in CRE bias.

LTE CRE parameter biasing by sigmoid mapping The cost of load balancing can be quite high in terms of handovers, and potentially also in terms of reduced link quality of a UE that is forced to connect to a cell other than the one with the best signal. For this reason, it is desirable to reserve the highest CRE settings for situations where the load balance is very bad. The mechanism of section 4.1.2 takes no account of this but simply tries to equalise the chosen metric by as large a range of CRE values as possible. An alternative is to scale the difference $b_{i}$ by means of a function that smoothly tapers off as it approach the end-points of the allowable range of CRE values. Sigmoid functions, such as the tangens hyperbolicus, turns out to be ideal for this purpose, especially if we augment them with a scaling constant that influences the steepness of the slope close to $b_{i}=0$. The function we use for this purpose is defined as follows:

$$
\mathcal{B}\left(b_{i},\left\{b_{j}\right\}_{j \in \mathcal{N}_{i}}, m, k\right)=m \frac{\tanh k b_{i}}{\tanh k}
$$

where $k$ is the scaling constant mentioned, and $m$ is a constant used to scale the $[-1,1]$ range of the tanh function to the allowable range of CRE bias values, i.e. 3 for $\pm 3 d B$. Although, in principle, the mean metric in the neighbourhood of a highly loaded cell can be close to one, the mean target will include a component of the target load of the current node, so the differences close to the extreme values of the $[-1,1] b_{i}$ value range are extremely rare. Figure 3 shows a plot for the function for $k=2$ and $m=6$.

Since we can arbitrarily choose how eagerly we scale the $b_{i}$ value to CRE settings, we can have anything from a linear mapping of $b_{i}$ values to CRE range( for $k \leq 1$ ) to a mapping which 


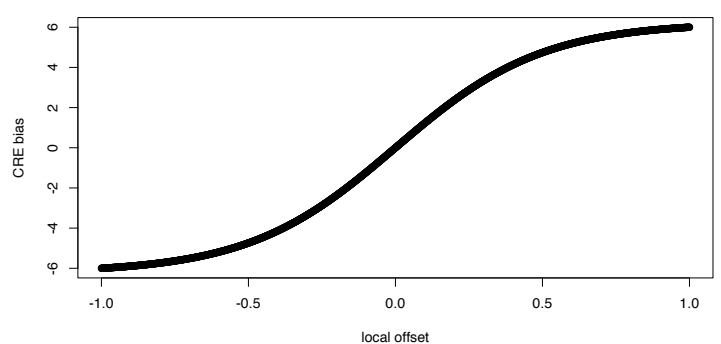

(a) $k=2, m=6$

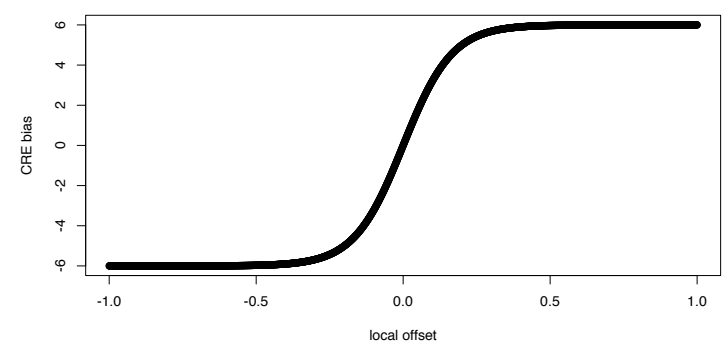

(b) $k=6, m=6$

Figure 3. Examples of two parameter settings for the sigmoid mapping of offset to CRE range

effectively reproduces the results of the mechanism of Section 4.1.2. Most interestingly perhaps, is that we can extend the allowable range of CRE values beyond the ones desirable in normal practice, and reserve the extreme values for cases where the load situation is such that the server with the best channel quality is so overloaded that connecting to it will provide no practical link quality advantage. Exactly at which point this occurs in real networks needs careful analysis of recorded data and practical experiments, but given such knowledge, scaling with sigmoids is a promising candidate to implement balancing policies. We will explore a few examples from the parameter range of Equation 11 in Section 5, but because of the simplicity of our radio model, these should be regarded as indicative, rather than conclusive.

\subsection{WiFi adaptation}

The load balancing method described in Section 4.1 was originally designed [1] for LTE, and even though it has not yet been evaluated outside that context, an analysis of the requirements of the method shows that it can in fact, quite easily be adopted to other RATs. E.g. in CDMA cellular networks, an almost identical method can be implemented with very similar effort, although exactly how the momentary measurements are obtained, and the communication between the nodes is implemented may differ slightly. The same goes for any future cellular system that implements a CRE type of mechanism.

In $\mathrm{WiFi}$, the IEEE 802.11 standard [30] and its amendments, including $802.11 \mathrm{r}, 802.11 \mathrm{k}$, and $802.11 \mathrm{v}$, support roaming within managed networks of access points (APs) served by one or more local controllers. These manage IP to MAC addresses translation, secure forwarding of connections, fast transition though pre-disconnection key exchange, radio state probing and link state sharing between APs, and assisted roaming though AP neighbour reports for clients and explicit transfer requests from both clients and APs.

The neighbourhood reports are based on collected RSSI probe statistics and configured AP topology and can be specialised for each AP and client. The combination of client neighbourhood reports and explicit transition requests gives the network a lot of control of the client roaming behaviour, but there is nothing stopping the client from spontaneously issuing transition requests, e.g. based on probes of the APs listed in the reports. The client roaming decision is not as standardised as in cellular, but even for clients without the assisted roaming extensions, transitions can be enforced by explicit disconnection and selective reconnection at more desirable APs.

This gives us several options for implementing our load balance proposal, but we will here only outline a version which is very similar to the one for LTE, and indicate the extensions required over existing mechanisms.

1. The $802.11 \mathrm{k}$ mechanism for generating neighbourhood reports based on RSSI probe statistics is extended to also generate local neighbourhoods for the APs based on transition statistics, as in Section 3.2.

2. The facility for sharing link state information between APs in $802.11 \mathrm{k}$ is extended with the metric and target value exchanges used in the algorithm of Section 3.4. Alternatively, metric values, or the statistics needed to compute them, are collected at the controller and used by 
a centralised version of the algorithm. The metrics used can be the same as, or variants of those proposed in Section 4.1.1.

3. Bias values are computed with one of the proposals in Section 4.1.2 and sent out to the clients via an extension of the $802.11 \mathrm{k}$ neighbourhood report

4. The clients actuate the rebalancing operation by spontaneously sending a $802.11 \mathrm{v}$ transition request whenever its RSSI+bias becomes less than that of a neighbouring AP, according the report. Alternatively, the request can be sent from the controller or the AP, preceded by a neighbourhood report indicating the desired target $\mathrm{AP}(\mathrm{s})$.

Implementing DTC for WiFi requires similar implementation effort as for LTE, but extensions of the existing standards are more extensive, and involves client changes.

Further steps towards coordinated resources in WiFi networks includes programmability frameworks. Recent proposals are based on the concept of light virtual APs (LVAP), abstracting the connection between the client and serving node, while simplifying state management following from client-AP (re-)association (e.g. [31-33]). The DTC approach could in this context, for example, serve the purpose of managing (by associated target values) the number of LVAP instances hosted per node, triggering instantiation or migration of LVAPs between serving nodes when needed. A full-fledged distributed implementation of DTC in a programmable framework would require local message exchanges in the control plane between neighbouring nodes (hosting control agents) for the purpose of updating target values, whereas a central control instance would set various parameters (e.g. actuator thresholds) influencing the overall behavior of DTC (see also Section 6.4).

\section{EXPERIMENTS}

To evaluate DTC in the RAN load balancing scenario under diverse conditions in a controlled and reproducible manner we have used simulations. We have examined the performance of several versions of the method in a wide range of scenarios. Each scenario repeats the user movement and traffic demand variations to compare versions of the method, and emulates the connections and traffic load of set of RAN nodes. The system model consists of the following components.

\subsection{Model system}

5.1.1. Traffic model The traffic model is based on a collection of random processes generating (downlink) traffic bursts. The simulator maintains one such process per simulated UE, where the rate of the burst arrivals is sampled from a lognormal distribution and the burst length and data rate are long-tailed (Pareto) variates. The parameters of the sampled distributions were fitted against recorded data of burst inter-arrival time (IAT), length and size obtained from WCDMA networks.

5.1.2. Mobility model The mobility model takes two essential aspects of user mobility into account, namely the distance between consecutive locations where the user resides for longer periods, and the fact that users - even though they occasionally make very long journeys - also tend to stay within a bounded area [34,35]. The model employed in the simulator is based on the observations made in [29], that the radius of gyration, $(\mathrm{RoG})$ i.e. the mean distance from a central point (CoG), and the locations a user visits over time can both be modelled by a truncated heavy-tailed distribution (THT), and that the flight lengths displayed by a user is strongly correlated with its RoG. Based on this observation, we sample heavy-tailed distances from a central position, which is randomly placed within the simulated area, but unique for each user, and an angle sampled from a uniform distribution to obtain goal positions for the next resting point. This results in heavy-tailed flight distances without excessively dispersing the users.

5.1.3. Radio model The radio model is based on two key components; a stochastic path loss calculation based on the model in [36], and a radio interference model developed by the authors. 
The path loss model is empirically based and emulate stochastic path loss per cell and UE location over a given distance, cell power and antenna placement in one of three geographical types.

To calculate the radio resource demand of a user with a given bit rate demand, and path loss, we estimate the signal to interference and noise ratio (SINR) at the users location. We assume the main components of this ratio to be the received signal power (RSP) of all (down link) transmissions, and calculate the noise and interference at a location as the sum of a fixed noise floor and the current RSP of all cells at the location. The output of the interfering cells is assumed to be cell power times the relative resource block utilisation averaged over a fixed time frame.

Handover decision are made based on smoothed down link RSP measurements, offset by the current value of the CRE parameter, and updated once per second. The contributions of each connected UE to the load of its connected cell is a function of the bandwidth demand prescribed by the traffic model, and the path loss at the UE's position.

\subsection{Simulation scenarios}

For each scenario, the placement and effective range of a given number and type of cells are fixed. The movement and traffic events for each UE are reproduced over each run of the scenario by using a fixed seed for the random generator used to sample inter-event times and magnitudes. The majority of UEs remain idle at any given time, but maintain their mobility and traffic demand simulations, in order to generate connection and handover events at realistic rates. Within each scenario, the effective range of each cell and path loss for each $5 \times 5$ meter area is determined by the samples of three random variables in the path-loss model. Two samples are chosen for each scenario and cell in addition to its placement and power, and one more is assigned to each area within a scenario.

\subsection{Collected statistics}

We consider the following set of statistics within each of 4 classes of scenarios with fixed area size overall, number of cells and UEs. Since placement and coverage of the cells vary between scenarios, we see a range of load levels and coverage between scenarios within a class. Each scenario is run for 30 simulated minutes, after a 3 minute run-in period. For each size class of scenario, we generate between 50 and 100 scenarios, and record for each time frame and scenario, the following statistics:

1. The mean load over the cells

2. The max load over the cells

3. The mean of the 95 th load $\%$-iles for each cell, measured as a running average

4. The corresponding max over the 95 th load \%-iles

5. The Jain fairness index of over the cells for intra cell running load mean and 95th \%-iles

6. The proportion of requested bits served

7. The number of hand-ins per connected user over all cells

where the Jain fairness index,

$$
J\left(x_{1}, \ldots, x_{n}\right)=\frac{\left(\sum_{i=1}^{n} x_{i}\right)^{2}}{n \sum_{i=1}^{n} x_{i}^{2}},
$$

measures the similarly of each metric (running load mean and 95th \%-ile), over the nodes $i$.

\subsection{Scenario classes}

We summarise these statistics with quantiles and means for each metric over all time frames and scenarios within each class, and vary the fixed area size, number of cells and user as follows

1. One macro and one smaller cell over an area of $2.25 \mathrm{~km}^{2}$ and 7000 UEs

2. One macro and two smaller cells over an area of $4.0 \mathrm{~km}^{2}$ and 8750 UEs

3. One macro and three smaller cells over an area of $6.25 \mathrm{~km}^{2}$ and 10500 UEs

4. Two macro cells and 4 smaller cells over an area of $9.0 \mathrm{~km}^{2}$ and 17500 UEs

5. Four macro cells and 11 smaller cells over an area of $27.0 \mathrm{~km}^{2}$ and 34900 UEs 


\subsection{Experimental setup}

Experiments are performed with several variants of the load balancing method engaged and compared to a base case with CRE set to 0 for all cells. The first of these is the load balancing method described in [1] (i.e. with load mean metric and maximal scaling), the second by mapping the distance between the actual and target loads to a given CRE range using the sigmoid as described in Section 4.1.2. The third and fourth replaces the load metric of the two previous methods with risk of exceeding $95 \%$ of the cell capacity as described in Section 4.1.2.

The LTE standard allows CRE values in the range $\pm 10 \mathrm{~dB}$, but in practice, the impact on the signal quality of individual UEs attached to a cell with less than about $6 \mathrm{~dB}$ below the strongest one, can be significant and should be avoided. This can be done in several ways, the simplest being a hard limit on the range of valid CRE values, but in principle we can also use methods that ensure that such situations remain rare. We will report results for a CRE range of $\pm 3 \mathrm{~dB}$ which guarantees that this will never happen, but also give examples for a CRE range of $\pm 6 \mathrm{~dB}$ where of impact of and differences between the methods are more clearly seen. For the sigmoid mechanism we will also report result on a $\pm 10 \mathrm{~dB}$ CRE range with CRE and RSP difference statistics.

\subsection{Results}

Let us have a look, first, at some general properties of the different classes of scenarios. Figure 4 shows box plots over the quantiles and mean of three metrics: 1) Mean and max momentary loads over the involved nodes, and 2) the proportion of the requested bits transferred. In this case, load means remain approximately constant over differently sized scenarios, while load max increase significantly and system efficiency goes down somewhat as the number of cells (and users) increases. In all cases, we see small but clear improvements for all the balancing methods with respect to the base line.



(a) Two-cell scenarios

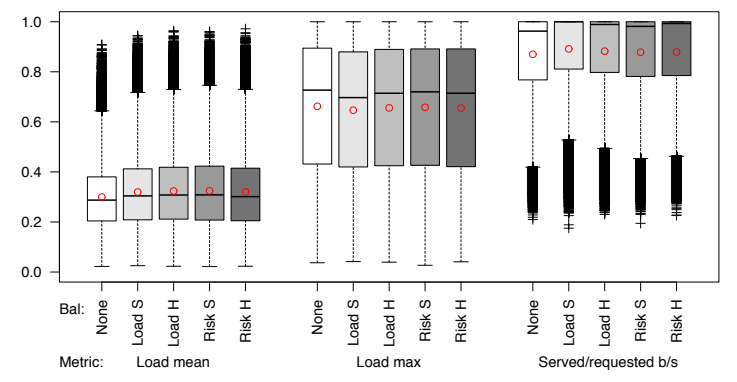

(b) Four-cell scenarios

Figure 4. General trends. Means over 100 scenarios, each lasting 30 minutes, \pm 3 dB CRE range.

The next set of graphs (Figure 5), show more clearly the impact of the various methods. Figure 5(a) shows the mean and max over the nodes of the 95th load \%-tile, while Figure 5(b) shows the corresponding Jain fairness index of the load means and 95th load \%-iles respectively. Note the strong reduction of the 95th \%-ile maximum, i.e. that of the highest loaded node, and the corresponding improvement of the Jain index, especially for the 95th \%-ile.

This improvement comes at the cost of an increased number of handovers. Figure 6(a) shows how the handover frequency more than doubles for the maximal scaling mechanism, while the cost for the risk balancing, in particular the one using the sigmoid mechanism, is more moderate.

5.6.1. Method comparisons Next we will see how the methods compare. Figure 7 shows the effect of each method relative to the base line for all metrics discussed above for the 15 cell, $34900 \mathrm{UE}$ case, and with a $\pm 6 \mathrm{~dB}$ CRE range. In Figure $7(\mathrm{a})$, we see that the mean load goes up by between $2 \%$ and $4 \%$, but that the max, i.e. the load of the highest loaded node, simultaneously goes down by between $1.5 \%$ and $3 \%$. The increase of the mean load is matched by a corresponding increase 

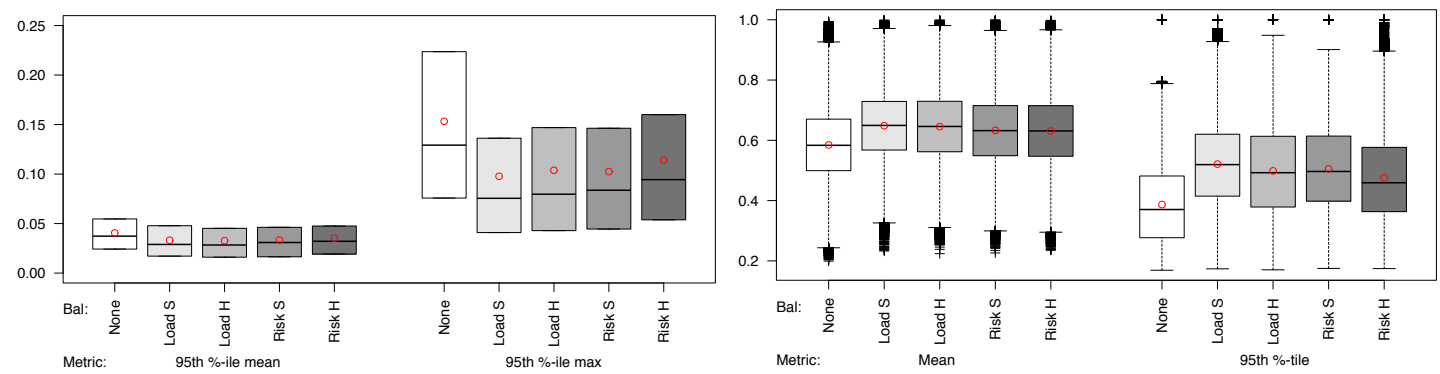

(a) Mean and max of the 95th \%-ile over the nodes

(b) Jain fairness index of mean and 95th \%-ile over the nodes.

Figure 5. 95:th \%-ile metrics. Means over 100 6-cell scenarios, 30 minute runs, \pm 3 dB CRE range.

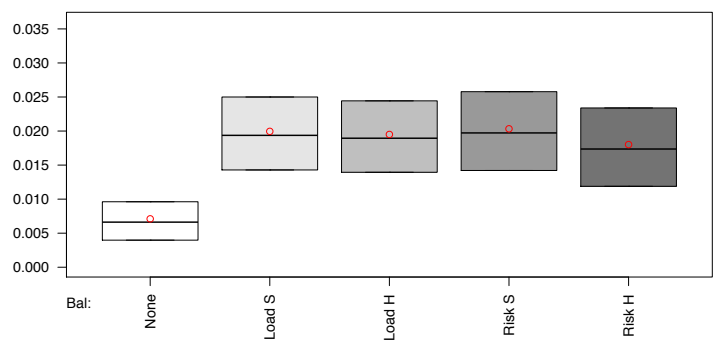

(a) 6-cell scenarios, $\pm 3 \mathrm{~dB}$ CRE range

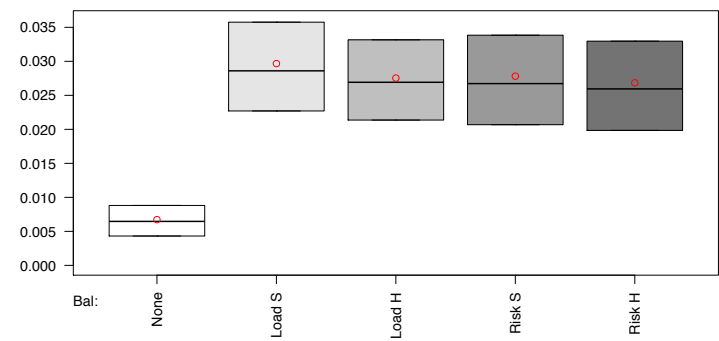

(b) 15-cell scenarios, $\pm 6 \mathrm{~dB}$ CRE range. High sigmoid scale

Figure 6. Handover freq. in handovers/s per connected user. Means over 100 and 50 scenarios, 30 min. runs

of served bits by between $4.1 \%$ and $6.5 \%$. Mean load fairness is improved by around .07 while the fairness of the really high loads (95:th load \%-ile) is increased by between .8 and .12 . In Figure 7(b), we see a slight decrease in mean of the 95th load \%-ile, but a decrease of around $8 \%$ in the 95 th $\%$-ile of the highest loaded node over all times and runs.

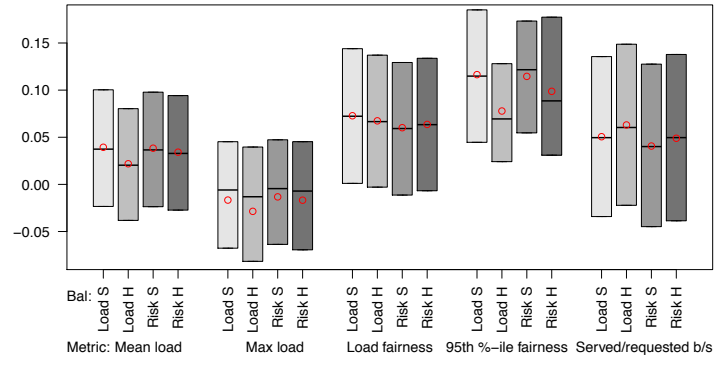

(a) Mean, max and fairness over nodes \& system throughput

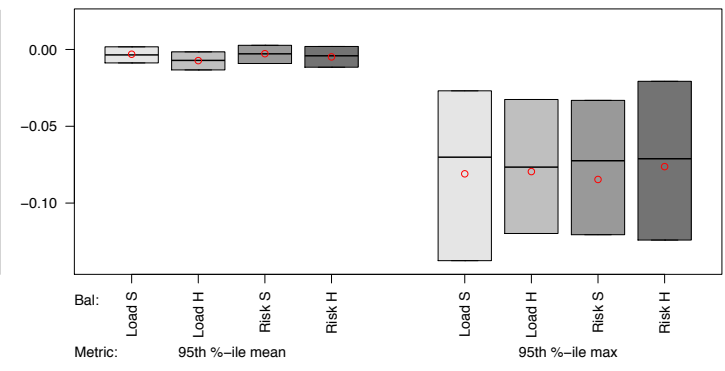

(b) Mean and max 95th \%-iles over nodes.

Figure 7. Relative improvement v.s. baseline. Means over 50 15-node- $30 \mathrm{~min}$. scenarios, \pm 6 dB CRE range.

These numbers are for a CRE range of $\pm 6 \mathrm{~dB}$ and the sigmoid parameters set to more or less match the performance of the maximal scaling mechanism. Looking at Figure 6(b), the cost in terms of handovers, shows an increase from $4 / \mathrm{min}$. for the base line to $>1.7 / \mathrm{min}$. for all balancing methods. Such numbers are probably not acceptable in practice, so we will next examine a more realistic case. The ones presented so far do show that 1) the level of high load reduction achievable by this type of method, and 2) the sigmoid mechanism can be parametrised to reproduce the performance of the maximal scaling mechanism, as long as we disregard the cost.

In Figure 8, we see the corresponding results for a CRE range of $\pm 3 \mathrm{~dB}$ and sigmoid parameters set to achieve a more reasonable balance between balancing performance and handover cost. In the Figure $8(\mathrm{a})$, we see a modest reduction of around $1 \%$ in the occurrence of high loads. The sigmoid 
scaling of the mean load (S) is most efficient at $1.3 \%$, but even the sigmoid scaling of the high load risk comes in at $0.5 \%$. The load fairness gives a detailed view, with improvements in Jain index values between .04 and .05 . The difference between the methods is most visible in the case of the Jain index over the running 95th load \%-ile which are improved by $0.27-0.53$. We can also see that the sigmoid scaling appears to improve system throughput more than the maximal scaling. In Figure 8(b) we see that, by adjusting the sigmoid parameters we can achieve comparable results to the maximal scaling but at a reduced cost in terms of handovers. Here the cost in handover frequency for the risk balancing with sigmoid scaling is only increased to $1.20 / \mathrm{min}$. from $.41 / \mathrm{min}$. for the baseline. Both sigmoid variants stay below $1.0 / \mathrm{min}$.



(a) Mean, max and fairness over nodes \& system throughput

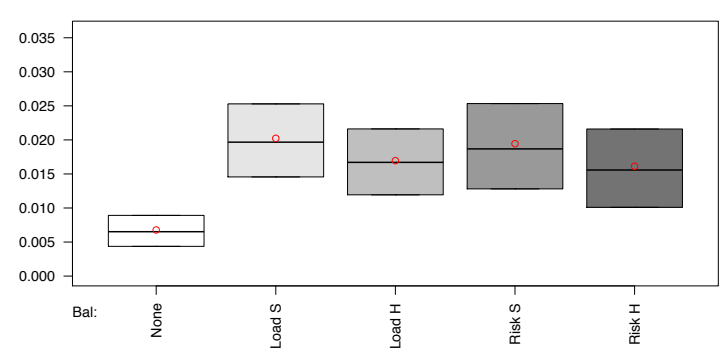

(b) Handover freq. in handovers/s per connected user.

Figure 8. Relative metrics v.s. base line. Means over 50 15-node-30 min. scenarios, $\pm 3 \mathrm{~dB}$ CRE range.

5.6.2. Sigmoid scaling with wide CRE range We will examine one more example where we reduce the sigmoid scale further, but at the same time increase the CRE range to that allowed by the LTE standard, i.e. $\pm 10 \mathrm{~dB}$. This means that there is a non-zero risk of an individual UE connecting to a node with a RSP up to $20 \mathrm{~dB}$ lower than the best one at a given location. In Figure 9(a)-9(d), we plot the distribution of differences between that of the node the UE is connected to, and that of the one with the strongest signal at the location of the UE for 50 scenarios of 3 nodes with low scale parameters and $\pm 10 \mathrm{~dB}$ limit, the sigmoids.

Since the individual handover decisions are actually based on a running estimate of the measured RSP value modulo the CRE offset values, the CRE difference is not directly translatable to RSP difference. We therefore also measured the resulting difference between the momentary RSP of the connected node, and that of the one with the strongest signal, with the result shown in Figure 9(e)9(h). It turns out that the probability of connecting to a node with a RSP lower, than the one with the strongest signal is actually lower using the sigmoid over the full $\pm 10 \mathrm{~dB}$ range, than using the maximal scaling mechanisms with an $\pm 3 \mathrm{~dB}$ range. The reason for this is that the maximal scaling forces UEs to connect to a non-optimal node even if the metric difference relatively is small, while the sigmoid uses large CRE values only when then metric difference is also large. Note that the bin containing the zero difference is truncated in the plots, and the absolute density values are very low. The the vast majority of UE-time frames, the UE connects to the strongest signal and reports a zero value. Note also, that both risk balancing methods reduce the impact compared to the mean load balancing ones.

\section{DISCUSSION}

\subsection{Experimental highlights}

It is clear from results of Section 5.6 that all versions of the proposed balancing method has significant and positive effect on the metrics examined, but there are also significant differences between the methods. Balancing the risk metric, as expected, achieves more balance between the 


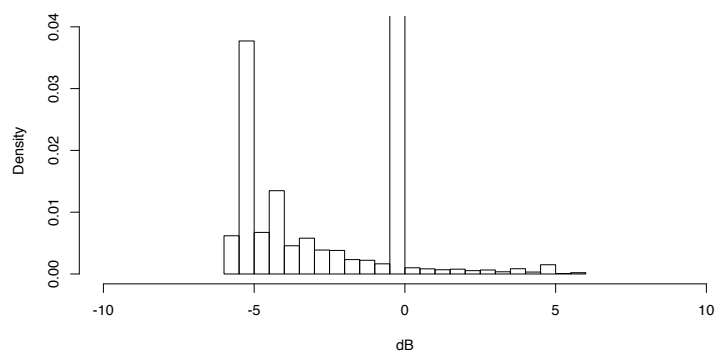

(a) CRE difference, balancing load

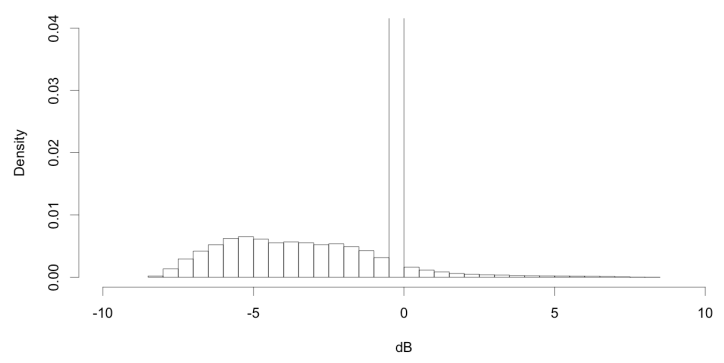

(b) CRE difference, balancing load with sigmoid

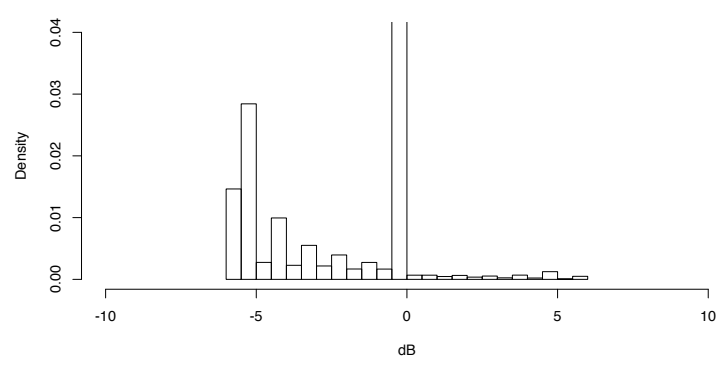

(c) CRE difference distribution, balancing risk

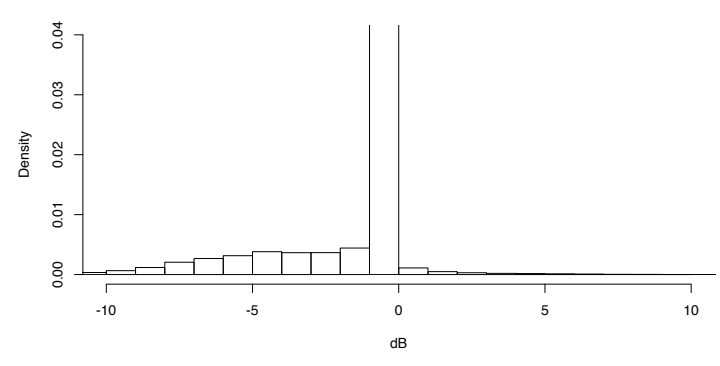

(d) CRE difference, balancing risk with sigmoid

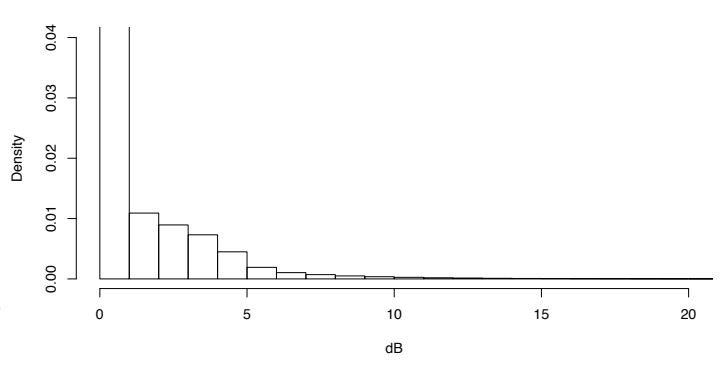

(e) RSP difference, balancing load

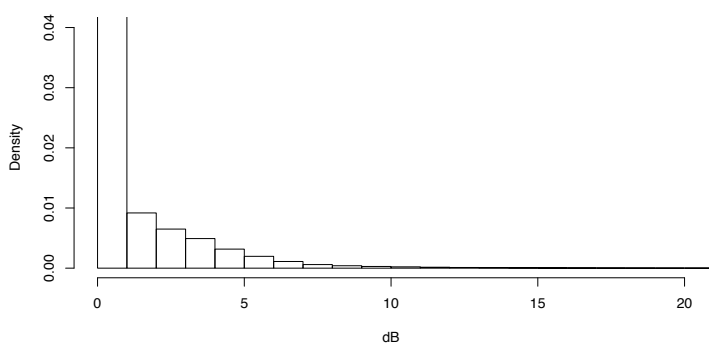

(f) RSP difference, balancing load with sigmoid



(g) RSP difference, balancing risk

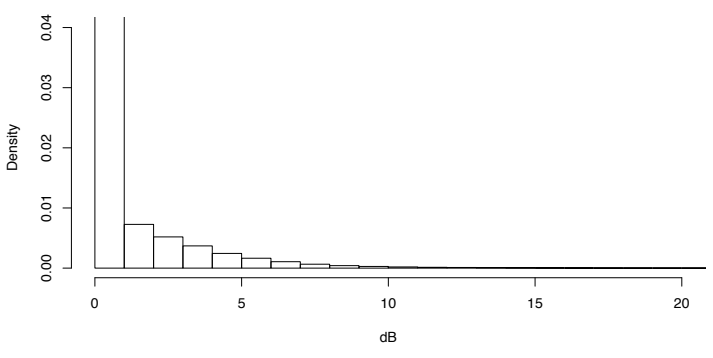

(h) RSP difference, balancing risk with sigmoid

Figure 9. CRE and RSP difference distributions for each method. Means over 50 3-node [30]min. scenarios of $\pm 10 \mathrm{~dB} C R E$ range for the sigmoids, and $\pm 3 \mathrm{~dB}$ for the others.

really high loads, as seen in the 95th \%-iles, and a bit less balance overall, but this is also reflected in lower cost in terms of handovers.

Highly significant reductions in handover cost can be achieved by using the sigmoid mapping, especially if we use low scaling factors and allow rare cases of extreme imbalance to result in occasional CRE offsets larger than $6 \mathrm{~dB}$. As indicated by Figure 9 the impact in terms of channel quality may not be so significant in practice. Finding practical sigmoid scaling factors and CRE ranges in a deployed RAN will require further experimentation. 


\subsection{Modelling considerations}

There is a risk that the load distributions will not turn out to be lognormal for every type of resource and RAN. In such cases the distribution may be modelled by other parametric distributions, or estimated empirically using quantiles. To the extent that the underlying demand distribution indeed is lognormal, a more thorough comparison between the modelled tail mass and measurements of the secondary mode (see Section 3) at high loads is also needed. The simplicity of the lognormal model, and the straightforward extraction of modelled quantiles proposed in Section 4.1.1 makes it attractive for many cases where the underlying process is even approximately lognormal.

\subsection{Implementation aspects across different RATs}

In Section 4.2, we outlined a version of the method for WiFi that is very similar to the one described for LTE in the context of intra-RAT load balancing. In practice, the implementation of DTC mechanisms and mobility management functions for both intra-RAT and inter-RAT operations are specific to the underlying technology and equipment. For intra-RAT operations, the implementation of the actuation mechanisms may therefore look very different depending on the RAT at hand. In cases of inter-RAT operations across multiple heterogeneous RATs, the key issue is to ensure that computed targets and biases are comparable which may require additional processing.

Even though we do not present any results for inter-RAT load balancing, we maintain that the algorithm is RAT-agnostic, due to the generality of the method and the use of probabilistic risk estimates favouring control in terms of high-level abstractions. In cases involving multiple and heterogeneous RATs, the level of implementation efforts varies with the diversity of the underlying technologies. In systems with similar RATs operating in line with more or less common standards and functionality, RAT-specific implementations should be relatively easy to adapt and modify with little or moderate efforts. Load balancing within a cellular system involving different generations of the same type of RAT is a typical example.

The expected densification of radio nodes needed for maintaining service availability in the future, requires that several technical challenges are solved for bridging the gap between heterogeneous infrastructures to achieve effective mobility operations. For example, the case of handoffs between cellular and WiFi comes with its own set of open problems, even though standards in this domain appear to be converging [37-39]. Once mechanisms for smooth handoffs between WiFi and cellular (and other RATs) are in place, the method proposed in this paper can be widely applied and extended for balancing one or multiple metrics within and across various RATs.

\subsection{Combining DTC and centralised control}

The formulation of the mechanism as a distributed algorithm enables a range of implementation possibilities from completely distributed, with only sparse monitoring information and control parameter adjustments passed between controllers and nodes, to a physically centralised solution where high resolution measurement are regularly collected and centrally managed. Regardless of the deployment strategy, monitoring data can be aggregated to a centralised monitoring function which is a highly useful feature inherent to the design of the method, offering great implementation and operational flexibility. Furthermore, the self-adjusting balancing method offers the right type of high-level policy parametrisation to fit into a centralised management scheme through the use of probabilistic metrics and thresholds. More specifically, the sigmoid scaling and range parameters, as well as the chosen load level for the risk estimates, are suitable candidates for a controller API enabling policy adjustments to shift balancing performance against handover and channel quality costs.

The overall idea of a logically centralised RAN control is effective coordination of radio resources. We believe that effective (i.e. timely and scalable) coordination of network resources can be realised in a distributed system that implements both logically centralised control functions while operating in a self-organising manner, where applicable. As an example of the combination of DTC and centralised management, we envision the proposed metrics and load balancing approach to be employed in a programmable RAN setting, involving a programmable infrastructure 
controlled by a logically centralised controller and a high-level framework for managing services and network operations (Figure 10). In this setting, estimated overload risks are communicated to the controller and management levels for sophisticated coordination and control of heterogeneous radio resources and parameters in relation to network operation and service requirements. For load balancing, actuator thresholds, scaling and range parameters are here communicated and set locally in the involved nodes and used for triggering RAT-specific and self-organised load balancing. In a logically centralised controller setting the probabilistic metric abstractions are necessary components for providing a unified network view while facilitating infrastructure configurations as well as communication between controllers for eventually self-organised inter-RAT operations.

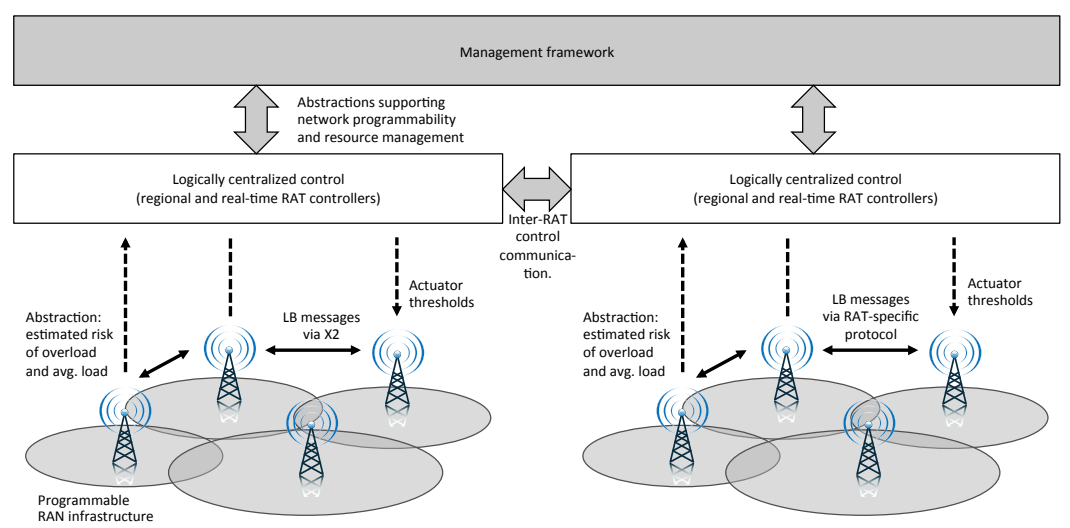

Figure 10. Conceptual overview of load balancing and corresponding abstractions in a programmable RAN.

\section{CONCLUSIONS}

We have described a generic method for dynamically balancing a given resource metric in distributed systems and exemplified its applicability in RANs with focus on load balancing in LTE and WiFi. The main features of the method include: simple deployment by the use of already existing mechanisms available in radio access technologies and standards; local and low complexity modeling; flexible implementation for both distributed and centralised management; probabilistic metrics and targets supporting RAT-agnostic operation and control.

The method has successfully been evaluated in a compound simulation environment based on a cellular RAN with path loss, traffic and mobility models. The results indicate that load balancing based on probabilistic overload risk metrics, compared to load balancing based on means, offers more robust solutions with the benefit of e.g. fewer handovers. The use of sigmoids for mapping target metrics to CRE bias values also provides a simple and convenient way of controlling the trade-off between balancing performance and cost.

The method is currently limited to a single metric and a future and useful extension would be the capability of balancing multiple metrics or composite targets. The applications enabled by such an extension would in a RAN setting entail balancing of e.g. backhaul and node compute capacity in addition to radio resources (e.g. for RAN-slicing), as well as flexible coverage patterns for radio access nodes. Finally, the general properties of the DTC approach open up for solving resource management problems also in wired networks, such as core networks and cloud systems, with applications ranging from routing, resource allocation and load balancing. 


\section{Acknowledgment}

This work was funded in part by the Swedish Foundation for Strategic Research (reference number RIT15-0075) and by the Commission of the European Union in terms of the 5G-PPP COHERENT project (Grant Agreement No. 671639).

\section{References}

1. Kreuger P, Görnerup O, Gillblad D, Lundborg T, Corcoran D, Ermedahl A. Autonomous load balancing of heterogeneous networks. 2015 IEEE 81st Vehicular Technology Conference (VTC Spring), 2015; 1-5, doi: 10.1109/VTCSpring.2015.7145712.

2. Kreuger P, Gillblad D, Görnerup O, Lundborg T, Corcoran D, Ermedahl A. Methods, nodes and system for enabling redistribution of cell load. Patent PCT/SE2014/050790 Jan 2016. Ericsson AB.

3. Gil J. Renaming and dispersing: Techniques for fast load balancing. J. Parallel and Distributed Computing November 1994; 23(2):149-158.

4. Bonomi F, Kumar A. Adaptive optimal load balancing in a nonhomogeneous multiserver system with a central job scheduler. IEEE Trans. Comput. October 1990; 39(10):1232-1250.

5. Kameda H, Li J, Kim C, Zhang Y. Optimal Load Balancing in Distributed Computer Systems. Springer Verlag: London, 1997.

6. Kim C, Kameda H. An algorithm for optimal static load balancing in distributed computer systems. IEEE Trans. Comput. March 1992; 41(3):381-384.

7. Hui C, Chanson S. Improved strategies for dynamic load balancing. IEEE Concurrency July-Sept 1999; 7(3):58-67.

8. Campos L, Scherson I. Rate of change load balancing in distributed and parallel systems. Parallel Computing July 2000; 26(9): 1213-1230.

9. Corradi A, Leonardi L, Zambonelli F. Diffusive load-balancing policies for dynamic applications. IEEE Concurrency Jan-March 1999; 7(1):22-31.

10. Chen XH. Adaptive traffic-load shedding and its capacity gain in cdma cellular systems. IEEE Proceedings Communications Jun 1995; 142(3):186-192, doi:10.1049/ip-com:19951913.

11. Bahl P, Hajiaghayi MT, Jain K, Mirrokni SV, Qiu L, Saberi A. Cell breathing in wireless lans: Algorithms and evaluation. IEEE Transactions on Mobile Computing 2007; 6(2):164-178.

12. Du L, Bigham J, Cuthbert LG, Nahi P, Parini C. Intelligent cellular network load balancing using a cooperative negotiation approach. WCNC, 2003.

13. Lobinger A, Stefanski S, Jansen T, Balan I. Load balancing in downlink LTE self-optimizing networks. Vehicular Technology Conference (VTC 2010-Spring), 2010 IEEE 71st, 2010; 1-5, doi:10.1109/VETECS.2010.5493656.

14. Siomina I, Yuan D. Load balancing in heterogeneous LTE: Range optimization via cell offset and loadcoupling characterization. Communications (ICC), 2012 IEEE International Conference on, 2012; 1357-1361, doi:10.1109/ICC.2012.6364075.

15. Wang H, Ding L, Wu P, Pan Z, Liu N, You X. Dynamic load balancing and throughput optimization in 3gpp LTE networks. Proceedings of the 6th International Wireless Communications and Mobile Computing Conference, IWCMC '10, ACM: New York, NY, USA, 2010; 939-943, doi:10.1145/1815396.1815611.

16. Hao W, Nan L, Zhihang L, Ping W, Zhiwen P, Xiaohu Y. A unified algorithm for mobility load balancing in 3gpp LTE multi-cell networks. Science China 2012; 1(12).

17. Foukas X, Nikaein N, Kassem MM, Marina MK, Kontovasilis K. FlexRAN: A Flexible and Programmable Platform for Software-Defined Radio Access Networks. Proceedings of the 12th International on Conference on Emerging Networking EXperiments and Technologies, CoNEXT '16, ACM: New York, NY, USA, 2016; 427-441, doi: $10.1145 / 2999572.2999599$.

18. Gudipati A, Perry D, Li LE, Katti S. Softran: Software defined radio access network. Proceedings of the second ACM SIGCOMM workshop on Hot topics in software defined networking, ACM, 2013; 25-30.

19. Kostopoulos A, Agapiou G, Kuo F, Pentikousis K, Cipriano A, Panaitopol D, Marandin D, Kowalik K, Alexandris $\mathrm{K}$, Chang C, et al.. Scenarios for 5g networks: The coherent approach. Telecommunications (ICT), $201623 \mathrm{rd}$ International Conference on, IEEE, 2016; 1-6.

20. Nunes BAA, Mendonca M, Nguyen XN, Obraczka K, Turletti T. A survey of software-defined networking: Past, present, and future of programmable networks. IEEE Communications Surveys \& Tutorials 2014; 16(3):1617-1634.

21. Kreuger P, Gillblad D, Arvidsson Å. Zero configuration adaptive paging (zCap). IEEE 76th Veh. Technol. Conf., IEEE, IEEE: Québec, 2012. URL http: / /www. ieeevtc. org/vtc2012fall/, 978-1-4673-1881-5.

22. Kreuger P, Gillblad D, Arvidsson $\AA$. zCap: A zero configuration adaptive paging and mobility management mechanism. Journal of Network Management 2013; 23:235-258.

23. 3GPP Technical Specification Group: Radio Access Network. X2 protocol. Technical Report TS36.423 (Release 12), 3GPP 2013.

24. Bishop CM. Pattern recognition. Machine Learning 2006; 128:1-58.

25. Fukuda K. Towards modeling of traffic demand of nodes in large scale network. Communications, 2008. ICC'08. IEEE International Conference on, IEEE, 2008; 214-218.

26. Downey AB. Lognormal and pareto distributions in the internet. Computer Communications 2005; 28(7):790-801.

27. Kreuger P, Steinert R. Scalable in-network rate monitoring. IFIP/IEEE Integrated Network Management - IM'15, IFIP/IEEE, IEEE: Ottawa, Canada., 2015.

28. Naboulsi D, Fiore M, Ribot S, Stanica R. Large-scale mobile traffic analysis: A survey. IEEE Communications Surveys and Tutorials 2016; 18:124-161. 
29. González MC, Barabási AL, Hidalgo CA. Understanding individual human mobility patterns. Nature June 2008; 453:779-782.

30. IEEE. 802.11: Wireless LAN Medium Access Control (MAC) and Physical Layer (PHY) Specifications. IEEE Standard 802.11, IEEE-SA 5 April 2012 revision, doi:10.1109/IEEESTD.2012.6178212.

31. Suresh L, Schulz-Zander J, Merz R, Feldmann A, Vazao T. Towards programmable enterprise wlans with odin. Proceedings of the first workshop on Hot topics in software defined networks, ACM, 2012; 115-120.

32. Schulz-Zander J, Mayer C, Ciobotaru B, Schmid S, Feldmann A. Opensdwn: Programmatic control over home and enterprise wifi. Proceedings of the 1st ACM SIGCOMM Symposium on Software Defined Networking Research, ACM, 2015; 16.

33. Riggio R, Rasheed T, Granelli F. Empower: A testbed for network function virtualization research and experimentation. Future Networks and Services (SDN4FNS), 2013 IEEE SDN for, IEEE, 2013; 1-5.

34. Song C, Koren T, Wang P, Barabási AL. Modelling the scaling properties of human moilbility. Nature Physics 2010; 6:818-823.

35. Song C, Qu Z, Blumm N, Barabási AL. Limits of predictability in human mobility. Science 2010; 327(5968):10181021.

36. Erceg V, Greenstein LJ, Tjandra SY, Parkoff SR, Gupta A, Kulic B, Julius AA, Bianchi R. An empirically based path loss model for wireless channels in suburban environments. IEEE Journal on Selected Areas in Communications July 1999; 17(7):1205-1211.

37. IEEE. 802.11u: Amendment 9: Interworking with External Networks. IEEE Standard 802.11, IEEE-SA 5 April 2011. URL http://standards.ieee.org/about/get/802/802.11.html.

38. IEEE. 802.21: Media Independent Handover. IEEE Standard 802.21, IEEE-SA 2015. URL http: //standards.ieee.org/about/get/802/802.21.html.

39. ETSI. Universal mobile telecommunications system (UMTS); LTE; IP flow mobility and seamless wireless local area network (WLAN) offload. TS 123 261, ETSI 2012. 